\title{
Review Article \\ Potential Challenges of Controlling Leishmaniasis in Sri Lanka at a Disease Outbreak
}

\author{
Tharaka Wijerathna, ${ }^{1}$ Nayana Gunathilaka, ${ }^{1}$ Kithsiri Gunawardana, ${ }^{1}$ and Wasana Rodrigo ${ }^{2}$ \\ ${ }^{1}$ Department of Parasitology, Faculty of Medicine, University of Kelaniya, Kelaniya, Sri Lanka \\ ${ }^{2}$ Biotechnology Unit, Industrial Technology Institute, Colombo 7, Sri Lanka \\ Correspondence should be addressed to Nayana Gunathilaka; n.gunathilaka@kln.ac.lk
}

Received 1 February 2017; Revised 30 March 2017; Accepted 30 April 2017; Published 28 May 2017

Academic Editor: Daniela Proverbio

Copyright (C) 2017 Tharaka Wijerathna et al. This is an open access article distributed under the Creative Commons Attribution License, which permits unrestricted use, distribution, and reproduction in any medium, provided the original work is properly cited.

\begin{abstract}
The present works reviewed the existing information on leishmaniasis in Sri Lanka and in other countries, focusing on challenges of controlling leishmaniasis in the country, in an outbreak. Evidence from recent studies suggests that there is a possibility of a leishmaniasis outbreak in Sri Lanka in the near future. Difficulty of early diagnosis due to lack of awareness and unavailability or inadequacy of sensitive tests are two of the main challenges for effective case management. Furthermore, the absence of a proper drug for treatment and lack of knowledge about vector biology, distribution, taxonomy and bionomics, and reservoir hosts make the problem serious. The evident potential for visceralization in the cutaneous variant of $L$. donovani in Sri Lanka may also complicate the issue. Lack of knowledge among local communities also reduces the effectiveness of vector and reservoir host control programs. Immediate actions need to be taken in order to increase scientific knowledge about the disease and a higher effectiveness of the patient management and control programs must be achieved through increased awareness about the disease among general public and active participation of local community in control activities.
\end{abstract}

\section{Background}

Leishmaniasis is a worldwide vector borne zoonotic disease caused by several heteroxenous intracellular parasitic organisms of genus Leishmania. The disease has several different forms including three main forms, namely, visceral leishmaniasis (VL), cutaneous leishmaniasis (CL), and mucocutaneous leishmaniasis (MCL), where VL is considered to be the most virulent form of the disease. Although several case reports provide evidences for sexual or vertical transmission [1-4], the main method of transmission of this disease is through the bite of the female sand flies of subfamily Phlebotominae.

Leishmaniasis has been listed as one of the eight major neglected tropical parasitic diseases with a prevalence in more than 90 countries where 88 of them are endemic to the disease. The zoonotic nature and the large genetic diversity of the both parasites and vectors make the control of this disease very difficult $[5,6]$.

In the control of leishmaniasis, early diagnosis and effective patient management, vector control, effective disease surveillance, control of reservoir hosts and social mobilization, and strengthening partnerships are the key strategies [6]. Early diagnosis and effective case management are essential to reduce the prevalence of the disease and prevent disabilities and death. There are no available vaccines or drugs to provide immunity to the infection. Therefore, the most effective way to prevent infection is prevention of sand fly bites by safety and vector control measures $[5,6]$.

\section{Leishmaniasis in Sri Lanka and Challenges in Controlling}

The first report of leishmaniasis from Sri Lanka was found in the 1990s. However, those were not due to local transmission, but reported mainly among overseas employees returning to the country [7]. First evidences for local transmission of leishmaniasis in Sri Lanka was in 1992 [8]. In 2008, this disease was named as a notifiable disease in Sri Lanka by the Epidemiology Unit of Sri Lanka [9]. Thereafter the disease was identified as a significant health problem in the country 
and a national action plan was adopted for the control of leishmaniasis [10]. All of the cases reported in past were cutaneous leishmaniasis. The first case of MCL was detected in 2005 [11] while the first patient with VL was identified 2007 [12]. At present, Sri Lanka is considered as a one of the endemic countries for CL [13] in the world and the newest reported focus of leishmaniasis in the Indian subcontinent where the disease is caused by the most virulent visceralizing species, Leishmania donovani. However, the potential for visceralization in the cutaneous variant of $L$. donovani in Sri Lanka is not known.

After 2001, an outbreak of CL in the island became evident, but still relatively an unknown disease [13]. However, VL and MCL have been reported less frequently than CL so far. The presence of vectors may increase the transmission risk and thereby the prevalence of the disease [14]. Studies have shown that the population of the vector species fluctuates with the seasonality (rainy or dry) and associated environmental factors [15]. In addition, the disturbances in environment which result in the loss of natural land coverage may also facilitate with favorable resting and breeding habitats for sand flies, thus increasing the probability of intensifying the sand fly population [14]. On the other hand, building constructions clearing original land coverage causes humans to get closer to living habitats of reservoir animals, further increasing the risk of infection. Increasing encroachment in to forest has resulted in such outbreaks in other countries $[14,16,17]$.Therefore, this disease can be more serious if a sudden outbreak of vector population occurred.

When considering the progress of the disease during last sixteen years, with increasing encroachment in to forests and ever-changing environmental conditions due to climate changes, occurrence of a serious outbreak of leishmaniasis in Sri Lanka is very likely. Hence, this review article emphasizes the potential challenges of controlling leishmaniasis in Sri Lanka at a probable outbreak and the steps that need to be taken in order to minimize adverse impact.

\section{Diagnosis of the Disease}

Early diagnosis is a very important factor for an effective management of any disease. Currently, several parasitological, molecular biological, and serological tests are used for the diagnosis. However, the diagnosis of leishmaniasis is still controversial as none of the available tests provide $100 \%$ specificity and sensitivity. Also the symptoms may vary from one form to another and also can be similar to some other diseases [18]. The simplest diagnostic method of leishmaniasis is microscopic identification of amastigote form of the parasite on a stained smears of samples obtained from skin lesions, liver, spleen, lymph nodes, or bone marrow $[10,19]$.

Advanced costly techniques such as Polymerase Chain Reaction (PCR) are more sensitive than microscopic observations [19-22]. Immunological methods are also widely used for the detection of parasites in blood. Mainly, Indirect Fluorescent Antibody (IFA) test is used to detect anti-leishmanial antibodies. Enzyme Linked Immunosorbent Assay (ELISA) is another serological test which is more useful in detecting visceral leishmaniasis. Immunochromatographic tests such as rK39 test which is based on recombinant K39 antigen are also performed in the detection of leishmaniasis $[13,23]$. In the diagnosis of CL, Leishmanin Skin Test (LST) can be used in order to measure delayed hypersensitivity caused by CL [24, 25]. Direct Agglutination Test (DAT) is relatively cheaper compared to other immunological tests mentioned above. However, this method is not appropriate since it has lower sensitivity $[10,19,20,22]$.

In Sri Lanka, the detection of the disease is passive and only the patients seeking medical attention for their symptoms are screened for the presence of parasites. No programs are conducted to detect patients at early stages of infection [13]. Detection mainly depends on microscopic observations of GIEMSA stained smears while advanced techniques such as PCR, ELISA, rK39, and other molecular diagnostic test are available in very limited laboratories $[13,26]$. These limitations of facilities to perform more advanced and sensitive tests can become an indispensable problem in early diagnosis, patient management, and prevention of the transmission of the disease.

Microscopic detection of parasites in VL requires some techniques that are very difficult to perform. For an example spleen aspiration which has a relatively higher sensitivity may be very dangerous since it can cause fatal damage to spleen and thus requires well trained personnel to perform $[19,27,28]$. Other samples that can be obtained easily from bone marrow and peripheral blood have a low sensitivity $[19,27]$. Although obtaining samples from skin lesions is much easier in CL, the results depend on the expertise of the observers and the quality of prepared slides [27]. Sensitivity can be improved by the culture of parasite, which in turn demands expensive facilities which is not available in a developing country like Sri Lanka and it is time consuming, thus reducing the effectiveness of the diagnosis [27, 29].

Other tests used in Sri Lanka, specially rK39 test, are inexpensive and rapid and can be performed by untrained person $[19,27,30]$ but less reliable as the sensitivity has been reported to be dependent on the ethnic group [29]. Therefore, this test may pose a considerable challenge in effective patient management of leishmaniasis in Sri Lanka especially in an outbreak situation.

\section{Treatments for the Disease}

Immunization for the disease is not yet established since the unavailability of a vaccine, although it appears to be very effective in controlling the disease [31]. As in all other neglected diseases, leishmaniasis also has the problem of having a minimum attention by drug developers since the treatment essentially needs to be affordable to poor population. Therefore, development of a vaccine cannot be expected in the near future. Hence, the control of the disease is mainly based on systemic and local treatments using drugs and/or nonpharmaceutical methods.

Systemic treatments include the administration of drugs through oral, intravenous (IV), intramuscular (IM), or intralymphatic (IL) routes [32]. Administration of Pentavalent antimonial drugs such as Sodium stibogluconate through 
IV and IM routes is the main method of the treatments despite the clinical representation of the disease [5, 33-35]. Administration of amphotericin B or Liposomal amphotericin B through IV route is also done in leishmaniasis treatments [36-39]. However, use of Liposomal amphotericin $\mathrm{B}$ considered as a better option than the conventional amphotericin B [5]. Oral administration of miltefosine and the IM administration of paromomycin and pentamidine are also used for leishmaniasis [37, 40-42]. In addition, certain azole compound normally used as antifungal agents including fluconazole and itraconazole are also used in treatments for both CL and MCL $[43,44]$.

In local or topical treatments for CL, both drugs and nonpharmaceutical agents are used. The drug that is mostly used in topical treatments is paromomycin, while some other drugs such as imidazole and methylbenzethonium chloride [45-47]. Use of some drugs in combination with other drugs showed to be more effective than using them alone. For an instance El-On et al. [48] show that the use of paromomycin sulfate in combination with methylbenzethonium chloride may be more effective in the treatment for CL lesions. Another study has also shown that the use of these two drugs with Gentamicin is significantly more effective than using previous two alone [49]. Intralesion administration of drugs such as Sodium stibogluconate and meglumine antimoniate is also used in pharmaceutical local treatments [50, 51]. Certain studies suggest that the intralesional administration of Sodium stibogluconate is more effective than the most common cryotherapy and has less side effects [50].

When considering nonpharmaceutical topical treatments, cauterization or the application of heat to the site of the lesion is the simplest treatment. This can be very effective since Leishmania parasites are heat sensitive [52, 53]. However, cryotherapy using liquid nitrogen is the most commonly used method [54].

In Sri Lanka, intralesional administration of Sodium stibogluconate is used as the main method of treatment for $\mathrm{CL}$, which is more common and known to have relatively less side effects [13]. However, practically it is not possible to use intralesional administration of drugs for all the situations. For an instance if the patient is a child and the lesion is at a sensitive part of the body, intralesional administration cannot be used. Then other methods such as heat treatments or cryotherapy should be used in based on the practical constraints. Another major problem is the high toxicity of drugs such as some antimonials used in treatments for all three forms of the disease. Any of the currently used antileishmanial drugs cannot be considered as the ideal drug for the disease due to high toxicity and the difficulty in administration $[55,56]$. On the other hand the resistance to the drugs used for treatments of leishmaniasis is evident from some studies, mainly for some pentavalent antimonial drugs $[40,57]$.

Other disadvantages of currently available drugs are the requirement of long term treatments, variation of efficacy from region to region, and the possibility of the recurrence of the disease $[32,34]$. On the other hand these treatments are not available in all the hospitals of the country. Patients must travel a long distance to those hospitals having dermatology clinics. Since most of these patients are farmers and daily workers, it creates considerable economic losses to their families. Therefore, the patient management will be a challenging task under these situations.

\section{Presence of Vectors and Vector Control Approaches}

Vector control is currently the most effective control strategy for leishmaniasis. Chemical, biological, and environmental methods are used to control leishmaniasis. When considering chemical control, indoor residual spraying is used worldwide. In Morocco, indoor residual spray has resulted in reducing disease incidence [58]. However, researches have shown that sand flies may develop resistance to these chemicals from both African and Asian countries [59, 60]. Therefore, main attention must be given toward environmental and biological control as well as prevention of bite of sand flies. Main method of environmental control is reducing the chance of breeding of sand flies altering environment to become unsuitable for breeding [61]. Normally sand flies breed in dark corners in the crevices of the walls having rich humus and moisture. Thus the most suitable way to control the disease is by preventing the breeding by plastering the walls [62] or by cement skirting on walls and the floor [63] which have experimentally proven to be effective in controlling sand fly populations. Certain biological control methods including the use of microorganisms to kill the larval stages are being tested and it has been proven to be effective, but the feasibility of practically use is yet to be evaluated [64-67].

However, controlling methods in many of the countries have been proposed and tested after thorough investigations on vector, for instance, exact vector species, behavior, breeding habits and places, resting places, distribution, and other bionomic characteristics such as population fluctuations. In Sri Lanka very few studies have been conducted on these aspects which is not sufficient to support for such control programs.

The most common vectors, Phlebotomine sand flies have a wide distribution in tropics and other warm mainland areas, and extend up to southern parts of the northern hemisphere [20]. There are more than 927 known species or subspecies of sand flies that have been identified so far around the world while only three genera, Phlebotomus, Lutzomyia, and Sergentomyia, are known to feed on vertebrate blood [20]. However, according to previous studies before 2014, only about 50 species of genera Phlebotomus and Lutzomyia are known to be responsible for the transmission of the disease to humans $[68,69]$, but complicating the issue even more, studies revealed that some species of genus Sergentomyia also have the ability to harbor Leishmania parasites suggesting a high likelihood of transmitting the disease by the species of the genus Sergentomyia [70, 71]. This is about to get worse as evidence is appearing to suggest that biting midges in the genus Culicoides also act as a host for the transmission of leishmaniasis [72]. However, the most common species of the genus, $P$. argentipes, is the proven vector of $L$. donovani which causes visceral leishmaniasis in the Indian subcontinent [73]. 
Presence of $P$. argentipes, the vector of $L$. donovani vector in other countries, has been reported from Sri Lanka in early 1940s [74]. Therefore, scientists suspected that $P$. argentipes is the vector of leishmaniasis in Sri Lanka. Interestingly, in 2009 studies revealed that all three members of Phlebotomus complex including, P. glaucus, $P$. argentipes, and P. annandalei are prevalent in Sri Lanka [75]. Molecular studies provide evidence for the presence of Leishmania DNA in blood fed and unfed female sand flies confirming the possibility of $P$. argentipes to act as vectors of leishmaniasis [76].

However, exact number of species that transmit the disease in Sri Lanka is yet poorly understood. Vector species range especially has been found to be broader than predicted earlier. For an instance, earlier only the genus Phlebotomus was known to act as vectors for Leishmania parasites, but as mentioned earlier, recent studies have proven the presence of Leishmania parasites in sand flies of the genus Sergentomyia. In Sri Lanka, 8 species from the genus Sergentomyia have been recorded [77-79]. Therefore, there is a possibility of these species acting as vectors in the country which is not yet discovered. Studies on biting midges and their potential capacity for the transmission of leishmaniasis also have rarely been studied in Sri Lanka. Hence, lack of knowledge on the species currently present from this group is a huge drawback as they apparently act as the vectors of leishmaniasis.

With the increasing complication of the issue, Sri Lanka has a huge challenge as the exact species that are transmitting the disease is yet unknown. On the other hand only a little knowledge is available on the species proven to be vectors in Sri Lanka, about their behavior, especially, breeding places, resting places, and the distribution which are essential in designing vector control strategies, and also there are no information on population dynamics of these sand flies which can be very useful in a sudden environmental change which may lead to an outbreak of sand flies and in turn of the disease, leishmaniasis.

In addition to control of sand fly populations, the prevention of sand fly bite is also important for effective control of the disease, for that also the information on biting times of sand flies and their habitat preferences which are very scanty for Sri Lankan species are needed.

\section{Availability of the Reservoir Hosts}

The infective agents of leishmaniasis are heteroxenous unicellular organisms where a single species of parasite can survive within several different hosts. Thus, the disease is a zoonosis with a range of sylvatic mammals, such as forest rodents, hyraxes, and wild canids and also domesticated animals mainly, dogs acting as reservoir hosts [80-85]. Reservoir hosts play an important role in the maintenance of the transmission cycle of the disease, because the cycle can be maintained by reservoir hosts, even in the absence of an infected human host [5]. Alterations in the range and density of the reservoirs, resulted from human made changes in the environment, may increase human exposure to infected sand flies [86]. Therefore, the zoonotic nature increases the prevalence of the disease.
Hence, the control and management of reservoir hosts is an effective control measure for leishmaniasis. For that the identification of exact species that act as reservoir hosts is essential. On the other hand their behavior, habitats, and specific habitat conditions are also needed to be understood in order to prevent the contact with humans. Screening of animal species individually for parasites is very difficult and time consuming. Hence, more effective methods such as screening blood fed sand flies for reservoir host DNA may give more reliable results in the identification of reservoir hosts $[87,88]$.

In Sri Lanka, few studies have been conducted to find out possible reservoir hosts for the disease and dogs have been identified as possible reservoir hosts $[81,89]$. However, some rodent species have been screened for parasites but the results have been negative according to the study conducted by Nawaratna et al. [81]. When considering the huge diversity of sylvatic and peridomestic mammals that have been reported from other countries as reservoir hosts for the disease, any mammal can be a reservoir, but except some rodents, no wild animals have been screened in previous studies in Sri Lanka as it is very difficult due to locate and catch these animals, especially bats, forest dwelling cryptic rodents, and the only wild canid in Sri Lanka, "the jackal" which has a possibility of acting as a reservoir host. Therefore, the reservoir host responsible for the prevalence of the disease may still remain unknown.

With the end of the civil war for 30 years, people have started to move in to previously uninhabited areas. The recent increase of the prevalence of the disease may have been partly influenced by these population movements. In these parasites which may have maintained their transmission cycle with a help of a reservoir host will infect humans when they are exposed to these. If the dogs are the only reservoir host for the disease, the control of stray dogs, screening and treatment of domestic dogs may provide a significant contribution to the control of leishmaniasis. The real problem arises if the reservoir host is a forest living animal as the control of this animal is difficult. The increasing deforestation and establishment of human habitations in recent past may also have a significant impact on the increased prevalence of the disease as this may take humans closer to reservoir hosts. Further, increase in these activities may cause a significant health issue as in the case of Iran during 1999-2001 where an outbreak resulted due to increased construction buildings near rodent colonies [16].

\section{Nature of the Parasite Species}

Intracellular parasites of the genus Leishmania of family Trypanosomatidae are the parasites of this disease. So far, about 21 species of this genus have been identified as causative agents of the disease including $L$. donovani complex with 2 species, $L$. donovani and $L$. infantum (or L. chagasi); $L$. mexicana complex with 3 main species, L. mexicana, $L$. amazonensis, and $L$. venezuelensis; L. tropica; L. major; $L$. braziliensis; L. guyanensis; L. panamensis; and L. peruviana [5]. Leishmania donovani complex is mainly responsible for the visceral form of the disease [19]. Out of these species 
L. donovani and L. tropica are the sources of anthroponotic infections, while most other species which cause cutaneous leishmaniasis act as sources of zoonotic infections. However, species of this complex also cause other types of the disease $[5,12]$.

In Sri Lanka L. donovani is the species that causes the cutaneous leishmaniasis [90]. The only strain isolated to date from skin lesions is Leishmania donovani MON-37, a zymodeme differing by only one nucleotide substitution in the glucose-6-phosphate dehydrogenase gene from $L$. donovani MON-2, the strain most commonly isolated from VL cases in India $[26,90]$. Therefore, Sri Lanka is considered as the newest reported focus of leishmaniasis in the Indian subcontinent where the disease is caused by the most virulent visceralizing species, L. donovani [9]. Hence, the case has become more serious as a potential for visceralization in the cutaneous variant of $L$. donovani in Sri Lanka beginning to appear.

\section{Lack of Proper Awareness about the Disease}

Awareness about the disease in local community is one of the main factors that determines the success of a control program. It is essential to have the knowledge on symptoms for effective case management. In order to prevent infection by following preventive measures such as reducing the contact with potential reservoir animals, preventing the bite of sand flies by reducing the exposed parts of the body during outdoor works, use of insecticide impregnated mosquito nets, and managing household environment to reduce the sand fly populations, people must know about how the disease get transmitted (reservoir hosts and vectors), the peak biting time of the vector, and how to prevent the bite and reduce the sand fly populations. Surveys have shown that the presence of adequate knowledge has significant contribution to the success of control programs through changes in behavior and more widespread participation [91, 92].

However, according to a recent survey conducted in an endemic area for the disease in Anuradhapura District of Sri Lanka shows that the level of awareness on this disease among local population is very low. For an instance, $37 \%$ of the study population have had never heard of the disease even they were living in a endemic region for the disease and nearly half of the study population had no clue about any advanced diagnostic and treatment facilities [93]. On the other hand, around $20 \%$ of people believe that sand fly bite has a mild or no effect for their health according to this study. Hence, inadequate knowledge and awareness on the disease by communities living in the endemic areas is one of the challenges for control and prevention efforts.

\section{Future Perspectives and Recommendations}

Leishmaniasis can be considered as a significant health issue at global level. Therefore, institutions such as World Health Organization are now focusing on development of advanced diagnostic tests, drugs, and vaccines [69]. An increase of the use of advanced molecular technologies [87, 88] and studies on biological control methods such as use of microorganisms, frogs, mites, and spiders as biological control agents can be seen in the recent past $[94,95]$.

Immunization for the disease is not available since there is no proper vaccine available in the market for leishmaniasis. On the other hand, none of the existing treatment methods are ideal as each method has any complication such as high toxicity, resistance by the parasite, or low efficacy.

Lack of adequate scientific knowledge and literature based on the research work is one of the main problems for the prevention and control of the disease in Sri Lanka. However, considerable improvement of research interest about leishmaniasis can be seen in recent years. But, the problem comes when applying these scientific knowledge effectively in the control processes. First of all steps must be taken to increase the awareness among local communities. In this case it can be very effective if these awareness programs can be integrated to the general health care programs.

In South Asian countries, despite the fact that these are male dominant societies, mothers can be considered as the Centre of the family; therefore, the target personnel must be mothers of families. School students are the next most important target group as it is easier to educate them. Provision of knowledge and getting the community participation in control efforts will increase their success effectively.

On the other hand, extended researches on the identification of vectors and reservoir hosts, their biology, distribution, bionomics, and other important factors must be encouraged. Use of advanced techniques in researches may improve the effectiveness of these researches. For an instance, in the identification of reservoir hosts, screening each potential species is less effective and highly impractical; therefore, advanced and more reliable methods such as sand fly blood meal identification where blood meals of blood fed sand flies are screened for the presence of host DNA use molecular biological techniques [88]. As mentioned above growing information suggests that sand flies are not the only vectors for the disease but also species of sand flies of the genus Sergentomyia which was known to be harmless and are proven to be potential vectors. Sexual transmission is also possible for this disease.

Therefore, studies must be focused more and more on these subjects for proper identification of exact vectors and other means of transmission in order to take preventive and control measures. In both vector and reservoir control activities much higher attention must be given to environmental management and biological control strategies other than the use of chemicals. Studies on environmental requirements of vectors and reservoir animals will provide information needed for control of vectors and reservoirs by reducing preferable conditions through environmental management. Several biological control strategies have been suggested by studies in other countries. These methods can be adopted to suit our country after feasibility studies.

Geographical Information System (GIS) is an effective tool that can create distribution maps of diseases. These maps can be used to understand geographical distribution patterns of the disease and associated risk factors. Although, use of GIS mapping has been shown to be very effective in disease control by the studies in other countries [14, 96, 97], in Sri 
Lanka GIS is not much popular as a disease control tool. It is possible to ameliorate the efficiency in decision making and planning activities in the control of leishmaniasis by promoting these techniques more and more.

Leishmaniasis also has some serious complications such as post-kala-azar dermal leishmaniasis (PKDL) which is a complication of $\mathrm{VL}$ that results in nodular rashes in patients recovered from VL $[73,98]$ and diffuse cutaneous leishmaniasis (DCL), which is believed to be a result of an immunological defect of the human host [99]. Early diagnosis and continuous surveillance are very important to prevent such complications.

Psychological studies suggest that depression and anxiety are significantly higher in leishmaniasis patients [100]. Therefore, it is also important to pay attention to postdisease psychological effects, in the case of CL, especially, which does not cause death or a significant morbidity but leaves a lifelong scar in exposed parts of the body which affects the normal lifestyle of the patients even when they completely healed.

\section{Conclusion}

Unavailability of immunization due to the absence of a proper vaccine and the absence of a completely safe and effective treatment method indicates the importance of the control of the disease through prevention of the infection. Major challenge for this is the lack of knowledge about disease. Sufficient information especially on vectors, reservoir hosts, and vector behavior in terms of biting time, resting preferences, breeding, oviposition behavior, and host range is not adequately documented. In addition, only few studies have been conducted to find out the exact reservoir host for the Leishmania parasites in Sri Lanka, such as screening dogs and some other rodent species while there is a large range of mammals which may have the ability to act as probable reservoir hosts that exist in Sri Lanka. Complexity of the action of parasite is also a challengeable aspect. Lack of awareness among local communities is also another drawback for the success of the control of the disease. On the other hand, not having the access to advanced diagnostic techniques may affect negatively on the effectiveness of case management. However, studies must be carried out to reveal the biology, distribution, bionomics, and other important characters of vectors and reservoir hosts. Awareness programs must be conducted to improve the advocacy of the general public on the disease in order to enhance community participation in control activities while promoting the use of advanced technologies and techniques for diagnosis and continuous surveillance of the disease.

\section{Abbreviations}

CL: Cutaneous leishmaniasis

MCL: Mucocutaneous leishmaniasis

VL: Visceral leishmaniasis

PCR: Polyclonal chain reaction

ELISA: Enzyme Linked Immunosorbent Assay

IFA: Indirect Fluorescent Antibody

DAT: Direct Agglutination Test
LST: Leishmanin Skin Test

PKDL: Post-kala-azar dermal leishmaniasis

DCL: Diffuse cutaneous leishmaniasis

IV: Intravenous

IM: Intramuscular

IL: Intralymphatic

GIS: Geographical Information System.

\section{Conflicts of Interest}

The authors have declared that they have no conflicts of interest.

\section{Authors' Contributions}

Tharaka Wijerathna and Nayana Gunathilaka wrote the review; all four authors read and approved the final version of the article.

\section{Acknowledgments}

The authors acknowledge financial support from the National Research Council, Sri Lanka, through Grant no. NRC 16-142.

\section{References}

[1] N. Aste, M. Pau, and P. Biggio, "Leishmaniasis of the prepuce," Journal of Europian Academy of Dermatology Venereology, vol. 16, no. 1, pp. 93-94, 2002.

[2] K. Kapila, M. B. Prakash, R. Mehrotra et al., "Testicular leishmaniasis in a boy with acute lymphoblastic leukemia," Acta Cytologica, vol. 38, no. 5, pp. 878-879, 1994.

[3] W. St. C. Symmers, "Leishmaniasis acquired by contagion. a case of marital infection in britain," The Lancet, vol. 275, no. 7116, pp. $127-132,1960$.

[4] M. S. Latrofa, F. D. Torres, D. D. Caprariis et al., "Vertical transmission of Anaplasma platys and Leishmania infantum in dogs during the first half of gestation," Parasites \& Vectors, vol. 9, article 269, 2016.

[5] Centre for Disease Control (CDC), “"Leishmaniasis”, 2013, http://www.cdc.gov/parasites/leishmaniasis.

[6] World Health Organization (WHO), “"Leishmaniasis”," 2016, http://www.who.int/mediacentre/factsheets/fs375/en/.

[7] T. D. Naotunne, S. Rajakulendran, W. Abeywickreme et al., "Cutaneous leishmaniasis in Sri Lanka. An imported disease linked to the middle east and african employment boom," Tropical and Geographical Medicine, vol. 42, no. 1, pp. 72-74, 1990.

[8] D. N. Athukorale, J. K. K. Seneviratne, R. L. Ihalamulla et al., "Locally acquired cutaneous leishmaniasis in Sri Lanka," The Journal of Tropical Medicine and Hygiene, vol. 95, no. 6, pp. 432433, 1992.

[9] Epidemiology Unit, Ministry of Health, and Sri Lanka, "Epidemiology of Leishmaniasis (Part II)," Weekly Epidemiology Report, vol. 37, no. 26, 2010.

[10] S. S. K. Nawaratna, D. J. Weilgama, C. J. Wijekoon, M. Dissanayake, and K. Rajapaksha, "Cutaneous leishmaniasis, Sri Lanka," Emerging Infectious Diseases, vol. 13, no. 7, pp. 10681070, 2007. 
[11] U. S. Rajapaksa, R. L. Ihalamulla, and N. D. Karunaweera, "First report of mucosal tissue localisation of leishmaniasis in Sri Lanka," Ceylon Medical Journal, vol. 50, no. 2, pp. 90-91, 2005.

[12] P. H. Abeygunasekara, Y. J. Costa, N. Seneviratne, N. Ratnatunga, and M. S. Wijesundera, "Locally acquired visceral leishmaniasis in Sri Lanka," The Ceylon Medical Journal, vol. 52, no. 1, pp. 30-31, 2007.

[13] H. Siriwardana, P. Chandrawansa, G. Sirimanna et al., "Leishmaniasis in Sri Lanka: a decade old story," Sri Lanka Journal of Infectious Diseases, vol. 2, no. 2, pp. 2-12, 2012.

[14] C. Ferro, M. López, P. Fuya, L. Lugo, J. M. Cordovez, and C. González, "Spatial distribution of sand fly vectors and eco-epidemiology of cutaneous leishmaniasis transmission in Colombia," PLoS ONE, vol. 10, no. 10, Article ID e0139391, 2015.

[15] E. F. de Oliveira, A. E. Casaril, W. S. Fernandes et al., "Monthly distribution of phlebotomine sand flies, and biotic and abiotic factors related to their abundance, in an urban area to which visceral leishmaniasis is endemic in Corumbá, Brazil," PLoS ONE, vol. 11, no. 10, Article ID e0165155, 2016.

[16] A. A. Akhavan, M. A. Yaghoobi-Ershadi, D. Mehdipour et al., "Epidemic outbreak of cutaneous leishmaniasis due to Leishmaniamajor in Ghanavat rural district," Iranian Journal of Public Health, vol. 32, no. 4, pp. 35-41, 2003.

[17] C. Ferro, D. Marín, R. Góngora et al., "Phlebotominae vector ecology in the domestic transmission of american cutaneous leishmaniasis in Chaparral, Colombia," The American Journal of Tropical Medicine and Hygiene, vol. 85, no. 5, pp. 847-856, 2011.

[18] M. L. Tierney and J. P. Mephee, Current Medical Diagnoses and Treatment of Leishmaniasis, Appleton and Language, Cambridge, Mass, USA, 1998.

[19] M. P. Barrett and S. L. Croft, "Management of trypanosomiasis and leishmaniasis," British Medical Bulletin, vol. 104, no. 1, pp. 175-196, 2012.

[20] G. Dawit, Z. Girma, and K. Simenew, "Review on Biology, epidemiology and public health significance of leishmaniasis," Journal of Bacteriology Parasitology, vol. 4, no. 2, Article ID 100166, 2013.

[21] G. Harms, G. Schonian, and H. Feldmeier, "Leishmaniasis in Germany," Emerging Infectious Diseases, vol. 9, no. 7, pp. 872$875,2003$.

[22] N. M. Ravasan, M. A. Oshaghi, E. Javadian et al., "Blood meal identification in field-captured sand flies: comparison of PCRRFLP and ELISA assay," Iranian Journal of Arthropod-Borne Diseases, vol. 3, no. 1, pp. 8-18, 2009.

[23] J. A. L. Lindoso, M. A. Cunha, I. T. Queiroz et al., "Leishmaniasis-HIV coinfection: current challenges," The Journal of HIV/AIDS-Research and Palliative Care, vol. 8, pp. 147-156, 2016.

[24] S. Singh, "New developments in diagnosis of leishmaniasis," The Indian Journal of Medical Research, vol. 123, no. 3, pp. 311-330, 2006.

[25] A. M. Musa, E. A. G. Khalil, M. A. Raheem et al., "The natural history of Sudanese post-kala-azar dermal leishmaniasis: clinical, immunological and prognostic features," Annals of Tropical Medicine and Parasitology, vol. 96, no. 8, pp. 765-772, 2002.

[26] N. D. Karunaweera, F. Pratlong, H. Y. Siriwardane et al., "Sri Lankan cutaneous leishmaniasis is caused by Leishmania donovani zymodeme MON-37," Transactions of the Royal Society of Tropical Medicine and Hygiene, vol. 97, no. 4, pp. 380-381, 2003.

[27] E. K. A. E. Elmahallawy, "Epidemiological Studies on Zoonotic Leishmaniasis and New Trials for Studying the Effect of Melatonin on the Parasite," 2015.
[28] P. Srivastava, A. Dayama, S. Mehrotra, and S. Sundar, "Diagnosis of visceral leishmaniasis," Transactions of the Royal Society of Tropical Medicine and Hygiene, vol. 105, no. 1, pp. 1-6, 2011.

[29] E. Abass, U. Steinhoff, and A. E. Harith, "Challenges in the diagnosis of visceral leishmaniasis in Sudan," International Journal of Collaborative Research on Internal Medicine and Public Health, vol. 8, no. 8, pp. 470-471, 2016.

[30] E. K. Elmahallawy, M. A. Sampedro, G. J. Rodriguez et al., "Diagnosis of leishmanis," Journal of Infection in Developing Countries, vol. 8, no. 8, pp. 961-972, 2014.

[31] L. Kedzierski, A. Sakthianandeswaren, J. M. Curtis, P. C. Andrews, P. C. Junk, and K. Kedzierska, "Leishmaniasis: current treatment and prospects for new drugs and vaccines," Current Medicinal Chemistry, vol. 16, no. 5, pp. 599-614, 2009.

[32] J. P. de Menezes, C. E. Guedes, A. L. Petersen, D. B. Fraga, and P. S. Veras, "Advances in development of new treatment for leishmaniasis," BioMed Research International, vol. 2015, Article ID 815023, 11 pages, 2015.

[33] P. L. Olliaro and A. D. M. Bryceson, "Practical progress and new drugs for changing patterns of leishmaniasis," Parasitology Today, vol. 9, no. 9, pp. 323-328, 1993.

[34] J. D. Berman, "Human leishmaniasis: clinical, diagnostic, and chemotherapeutic developments in the last 10 years," Clinical Infectious Diseases, vol. 24, no. 4, pp. 684-703, 1997.

[35] L. H. Freitas-Junior, E. Chatelain, H. A. Kim, and J. L. SiqueiraNeto, "Visceral leishmaniasis treatment: what do we have, what do we need and how to deliver it?" International Journal for Parasitology: Drugs and Drug Resistance, vol. 2, pp. 11-19, 2012.

[36] S. L. Croft and V. Yardley, "Chemotherapy of leishmaniasis," Current Pharmaceutical Design, vol. 8, no. 4, pp. 319-342, 2002.

[37] S. L. Croft and G. H. Coombs, "Leishmaniasis-current chemotherapy and recent advances in the search for novel drugs," Trends in Parasitology, vol. 19, no. 11, pp. 502-508, 2003.

[38] S. L. Croft, K. Seifert, and V. Yardley, "Current scenario of drug development for leishmaniasis," Indian Journal of Medical Research, vol. 123, no. 3, pp. 399-410, 2006.

[39] S. L. Croft and P. Olliaro, "Leishmaniasis chemotherapy-challenges and opportunities," Clinical Microbiology and Infection, vol. 17, no. 10, pp. 1478-1483, 2011.

[40] S. Sundar, "Drug resistance in Indian visceral leishmaniasis," Tropical Medicine and International Health, vol. 6, no. 11, pp. 849-854, 2001

[41] D. Fong, M. M.-Y. Chan, R. Rodriguez, L. J. Gately, J. D. Berman, and M. Grogl, "Paromomycin resistance in Leishmania tropica: lack of correlation with mutation in the small subunit ribosomal RNA gene," American Journal of Tropical Medicine and Hygiene, vol. 51, no. 6, pp. 758-766, 1994.

[42] A. Jhingran, B. Chawla, S. Saxena, M. P. Barrett, and R. Madhubala, "Paromomycin: uptake and resistance in Leishmania donovani," Molecular and Biochemical Parasitology, vol. 164, no. 2, pp. 111-117, 2009.

[43] J. Consigli, C. Danielo, V. Gallerano, M. Papa, and A. Guidi, "Cutaneous leishmaniasis: successful treatment with itraconazole," International Journal of Dermatology, vol. 45, no. 1, pp. 46-49, 2006.

[44] A. A. Alrajhi, E. A. Ibrahim, E. B. De Vol, M. Khairat, R. M. Faris, and J. H. Maguire, "Fluconazole for the treatment of cutaneous leishmaniasis caused by Leishmania major," The New England Journal of Medicine, vol. 346, no. 12, pp. 891-895, 2002.

[45] J. El-On and G. Messer, "Leishmania major: antileishmanial activity of methylbenzethonium chloride," American Journal of Tropical Medicine and Hygiene, vol. 35, no. 6, pp. 1110-1116, 1986. 
[46] B. A. Arana, C. E. Mendoza, N. R. Rizzo, and A. Kroeger, "Randomized, controlled, double-blind trial of topical treatment of cutaneous leishmaniasis with paromomycin plus methylbenzethonium chloride ointment in Guatemala," American Journal of Tropical Medicine and Hygiene, vol. 65, no. 5, pp. 466-470, 2001.

[47] S. Brenner, "Topical treatment with imidazole for cutaneous leishmaniasis," Israel Journal of Medical Sciences, vol. 21, no. 2, pp. 163-164, 1985.

[48] J. El-On, G. P. Jacobs, E. Witztum, and C. L. Greenblatt, "Development of topical treatment for cutaneous leishmaniasis caused by Leishmania major in experimental animals," Antimicrobial Agents and Chemotherapy, vol. 26, no. 5, pp. 745-751, 1984.

[49] K. C. Carter, J. Alexander, and A. J. Baillie, "Studies on the topical treatment of experimental cutaneous leishmaniasis: The therapeutic effect of methyl benzethonium chloride and the aminoglycosides, gentamicin and paromomycin," Annals of Tropical Medicine and Parasitology, vol. 83, no. 3, pp. 233-239, 1989.

[50] M. S. Gurei, N. Tatli, H. Ozbilge et al., "Efficacy of cryotherapy and intralesional pentostam in treatment of cutaneous leishmaniasis," Journal of the Egyptian Society of Parasitology, vol. 30, no. 1, pp. 169-176, 2000.

[51] P. Layegh, O. Rajabi, M. R. Jafari et al., "Efficacy of topical liposomal amphotericin B versus intralesional meglumine antimoniate (glucantime) in the treatment of cutaneous leishmaniasis," Journal of Parasitology Research, vol. 2011, Article ID 656523, 5 pages, 2011.

[52] J. D. Berman and F. A. Neva, "Effect of temperature on multiplication of Leishmania amastigotes within human monocytederived macrophages in vitro," American Journal of Tropical Medicine and Hygiene, vol. 30, no. 2, pp. 318-321, 1981.

[53] D. L. Sacks, A. Barral, and F. A. Neva, "Thermosensitivity patterns of Old vs. new world cutaneous strains of Leishmania growing within mouse peritoneal macrophages in vitro," American Journal of Tropical Medicine and Hygiene, vol. 32, no. 2, pp. 300-304, 1983.

[54] V. Leibovici and H. Aram, "Cryotherapy in Acute Cutaneous Leishmaniasis," International Journal of Dermatology, vol. 25, no. 7, pp. 473-475, 1986.

[55] V. S. Amato, F. F. Tuon, and A. M. Siqueira, "Treatment of mucosal leishmaniasis in latin america: systematic review," The American Journal of Tropical Medicine and Hygiene, vol. 77, no. 2, pp. 266-274, 2007.

[56] J. van Griensven, M. Balasegaram, F. Meheus, J. Alvar, L. Lynen, and M. Boelaert, "Combination therapy for visceral leishmaniasis," The Lancet Infectious Diseases, vol. 10, no. 3, pp. 184-194, 2010.

[57] F. Jeddi, R. Piarroux, and C. Mary, "Antimony resistance in Leishmania, focusing on experimental research," Journal of Tropical Medicine, vol. 2011, Article ID 695382, 15 pages, 2011.

[58] C. Faraj, E. B. Adlaoui, S. Ouahabi et al., "Field evaluation of alphacypermethrin in indoor residual spraying for leishmaniasis control in an endemic area, northern Morocco," Parasites and Vectors, vol. 6, no. 1, article 354, 2013.

[59] M. M. Hassan, S. O. Widaa, O. M. Osman et al., "Insecticide resistance in the sand fly, Phlebotomus papatasi from Khartoum State, Sudan," Parasites and Vectors, vol. 5, article 46, 2012.

[60] R. Singh, R. K. Das, and S. K. Sharma, "Resistance of sand flies to DDT in Kala-azar endemic districts of Bihar, India," Bulletin of the World Health Organization, vol. 79, no. 8, p. 793, 2001.
[61] K. Kishore, V. Kumar, S. Kesari et al., "Vector control in leishmaniasis," Indian Journal of Medical Research, vol. 123, no. 3, pp. 467-472, 2006.

[62] V. Kumar, D. K. Kesari, N. K. Sinha et al., "Field trial of an ecological approach for the control of Phlebotomus argentipes using mud and lime plaster," The Indian Journal of Medical Research, vol. 101, pp. 154-156, 1995.

[63] R. C. Dhiman, "Effect of minor engineering intervention in the control of breeding of Phlebotomus papatasi (Scopoli) sandflies," The Southeast Asian Journal of Tropical Medicine and Public Health, vol. 26, no. 2, pp. 368-370, 1995.

[64] L. L. Robert, M. J. Perich, Y. Schlein et al., "Phlebotomine sand fly control using bait-fed adults to carry the larvicide Bacillus sphaericus to control the larval habitat," Journal of the American Mosquito Control Association, vol. 13, no. 2, pp. 140-144, 1997.

[65] L. L. Robert, M. J. Perich, Y. Schlein et al., "Bacillus sphaericus inhibits hatching of sand fly eggs," Journal of the American Mosquitoe Control Association, vol. 14, no. 3, pp. 351-352, 1998.

[66] Y. Schlein, "Marking of Phlebotomus papatasi (Diptera: Psychodidae) by feeding on sprayed, coloured sugar bait: a possible means of behavioral and control studies," Transactions of the Royal Society of the Tropical Medicine and Hygiene, vol. 81, no. 4, p. 599, 1987.

[67] B. Yuval and A. Warburg., "Susceptibility of adult phlebotomine sand flies (Diptera: Psychodidae) to Bacillus thuringiensis var. israelensis," Annals of Tropical Medicine and Parasitology, vol. 195, no. 2, pp. 195-196, 1989.

[68] A. U. Bari and S. B. Rahman, "Cutaneous leishmaniasis: an overview of parasitology and host-parasite-vector inter relationship," Journal of Pakistan Association of Dermatologists, vol. 18, no. 1, pp. 42-48, 2008.

[69] P. Desjeux, "Leishmaniasis: current situation and new perspectives," Comparative Immunology Microbiology and Infectious Diseases, vol. 27, no. 5, pp. 305-318, 2004.

[70] K. Jaouadi, W. Ghawarl, S. Salem et al., "First report of naturally infected Sergentomyia minuta with Leishmania major in Tunisia," Parasites and Vectors, vol. 8, article 649, 2015.

[71] C. O. Nzelu, H. Kato, N. Puplampu et al., "First detection of Leishmania tropica DNA and trypanosoma species in Sergentomyia sand flies (diptera: psychodidae) from an outbreak area of cutaneous leishmaniasis in Ghana," PLoS Neglected Tropical Diseases, vol. 8, no. 2, article e2630, 2014.

[72] J. M. M. Rebêlo, B. L. Rodrigues, M. D. C. A. Bandeira et al., "Detection of Leishmania amazonensis and Leishmania braziliensis in Culicoides (Diptera, Ceratopogonidae) in an endemic area of cutaneous leishmaniasis in the Brazilian Amazonia," Journal of Vector Ecology, vol. 41, no. 2, pp. 303-308, 2016.

[73] C. S. Swaminath, H. E. Shortt, and L. A. Anderson, “Transmission of Indian kala-azar to man by the bite of $P$. argentipes ann and brun. 1942," The Indian Journal of Medical Research, vol. 123, no. 3, pp. 473-477, 2016.

[74] D. J. Lewis and R. Killik Kendrik, "Some phlebotomid sand flies of Malaysia and Sri Lanka," Transactions of the Royal Society of Tropical Medicine and Hygiene, vol. 67, no. 4, pp. 68-70, 1973.

[75] S. Ranasinghe, R. D. C. Maingon, and D. P. Bray, "A morphologically distinct Phlebotomus argentipes population from active cutaneous leishmaniasis foci in central Sri Lanka," Memorias do Instituto Oswaldo Cruz, Rio de Janeiro, vol. 107, no. 3, pp. 402409, 2012.

[76] K. Gajapathy, L. B. S. Peiris, and S. L. Goodacre, "Molecular identification of potential leishmaniasis vector species within 
the Phlebotomus (Euphlebotomus) argentipes species complex in Sri Lanka," Parasites and Vectors, vol. 6, no. 1, article 302, 2013.

[77] K. Gajapathy and S. N. Surendran, "Morphometric description of Sand flies belong to Genus Sergentomyia (Sergentomyia) (Diptera; Psychodidae; Phlebotominae) in Sri Lanka; evidence for the presence of Sergentomyia (Sergentomyia) pondicherriensis \&amp; Sergentomyia (Sergentomyia) dentata in the Country," Journal of Entomology, vol. 8, no. 6, pp. 512-529, 2011.

[78] Y. Ozbel, C. Sanjoba, B. Alten et al., "Distribution and ecological aspects of sand fly (Diptera: Psychodidae) species in Sri Lanka," Journal of Vector Ecology, vol. 36, 1, pp. S77-S86, 2011.

[79] T. Tharmatha, K. Gajapathy, R. Ramasamy et al., "Morphological and molecular identification of cryptic species in the sergentomyia bailyi (Sinton, 1931) complex in Sri Lanka," Bulletin of Entomological Research, vol. 107, no. 1, pp. 58-65, 2016.

[80] S. P. Brandão-Filho, M. E. Brito, F. G. Carvalho et al., "Wild and synanthropic hosts of Leishmania (Viannia) braziliensis in the endemic cutaneous leishmaniasis locality of Amaraji, Pernambuco State, Brazil," Transactions of the Royal Society of Tropical Medicine and Hygiene, vol. 97, no. 3, pp. 291-296, 2003.

[81] S. S. K. Nawaratna, D. J. Weilgama, and K. Rajapaksha, "Cutaneous leishmaniasis in Sri Lanka: a study of possible animal reservoirs," International Journal of Infectious Diseases, vol. 13, no. 4, pp. 513-517, 2009.

[82] J. B. Nunes, M. D. Laurenti, and H. Y. Kanamura, "Leishmania infantum infection in dogs from southern region of Minas Geais State, Brazil," Revista do Instituto de Medicina Tropical de Sao Paulo, vol. 58, article 75, 2016.

[83] D. Piantedosi, V. Veneziano, T. D. Muccio et al., "Epidemiological survey on Leishmania infection in red foxes (Vulpes vulpes) and hunting dogs sharing the same rural area in Southern Italy," Acta Parasitologica, vol. 61, no. 4, pp. 769-775, 2016.

[84] J. A. G. Silveira, M. L. D’Elia, I. D. O. Avelar et al., "Rangelia vitalii in a free-ranging maned wolf (Chrysocyon brachyurus) and co-infections," International Journal for Parasitology: Parasites and Wildlife, vol. 5, no. 3, pp. 280-285, 2016.

[85] U. Sharma and S. Singh, "Insect vectors of Leishmania: distribution, physiology and their control," Journal of Vector Borne Diseases, vol. 45, no. 4, pp. 255-272, 2008.

[86] Z. Assimina, K. Charilaos, and B. Fotoula, "Leishmaniasis: an overlooked public health concern," Health Science Journal, vol. 2, no. 4, pp. 196-205, 2008.

[87] M. Baum, E. A. D. Castro, M. C. Pinto et al., "Molecular detection of the blood meal source of sand flies (Diptera:Psychodidae) in a transmission area of American cutaneous leishmaniasis, Paraná State, Brazil," Acta Tropica, vol. 143, pp. 8-12, 2015.

[88] K. G. D. S. Sales, P. L. Costa, R. C. S. D. Morais et al., "Identification of phlebotomine sand fly blood meals by realtime PCR," Parasites and Vectors, vol. 8, article 230, 2015.

[89] A. C. Rosypal, S. Tripp, C. Kinlaw et al., "Surveillance for antibodies to Leishmania spp. in dogs from Sri Lanka," Journal of Parasitology, vol. 96, no. 1, pp. 230-231, 2010.

[90] H. V. Y. D. Siriwardana, H. A. Noyes, N. J. Beeching, M. L. Chance, N. D. Karunaweera, and P. A. Bates, "Leishmania donovani and cutaneous leishmaniasis, Sri Lanka," Emerging Infectious Diseases, vol. 13, no. 3, pp. 476-478, 2007.

[91] A. Alemi, A. Alemu, N. Esmael et al., "Knowledge, attitude and practices related to visceral leishmaniasis among residents in Addis Zemen town, South Gondar, Northwest Ethiopia," BMC Public Health, vol. 13, article 382, 2013.
[92] B. K. A. Borges, J. A. Silva, J. P. A. Haddad et al., "Assessment of knowledge and preventive attitudes concerning visceral leishmaniasis in Belo Horizonte," Cadernos de Saúde Pública, vol. 24, no. 4, pp. 777-784, 2008.

[93] S. Ranasinghe, R. Wickremasinghe, A. Munasinghe et al., "Cross-sectional study to assess risk factors for leishmaniasis in an endemic region in Sri Lanka," The Amrican Journal of Tropical Medicine and Hygiene, vol. 89, no. 4, pp. 742-749, 2013.

[94] D. S. Dinesh, V. Kumar, S. Kesari, and P. Das, "Mites and spiders act as biological control agent to sand flies," Asian Pacific Journal of Tropical Disease, vol. 4, supplement 1, pp. S463-S466, 2014.

[95] J. R. Khatiwad, S. Ghimire, S. P. K. Paudel et al., "Frogs as potential biological control agents in the rice fields of Chitwan, Nepal," Agriculture, Ecosystems and Environment, vol. 230, pp. 307-314, 2016.

[96] F. Abedi-Astaneh, H. Hajjaran, M. R. Yagoo bi-Ershadi et al., "Risk mapping and situational analysis of cutaneous leishmaniasis in an endemic area of central iran: a gis-based survey," PLoS ONE, vol. 18, no. 8, Article ID e0161317, 2016.

[97] R. Gálvez, M. A. Descalzo, I. Guerrero, G. Miró, and R. Molina, "Mapping the current distribution and predicted spread of the leishmaniosis sand fly vector in the Madrid Region (Spain) based on environmental variables and expected climate change," Vector-Borne and Zoonotic Diseases, vol. 11, no. 7, pp. 799-806, 2011.

[98] E. E. Zijlstra, A. M. Musa, E. A. G. Khalil, I. M. El Hassan, and A. M. El-Hassan, "Post-kala-azar dermal leishmaniasis," Lancet Infectious Diseases, vol. 3, no. 2, pp. 87-98, 2003.

[99] J. Convit, M. Ulrich, M. A. Polegre et al., “Therapy of Venezuelan patients with severe mucocutaneous or early lesions of diffuse cutaneous leishmaniasis with a vaccine containing pasteurized Leishmania promastigotes and bacillus Calmette-Guerin: preliminary report," Memorias do Instituto Oswaldo Cruz, Rio de Janeiro, vol. 99, no. 1, pp. 57-62, 2004.

[100] M. Yanik, M. S. Gurel, Z. Simsek, and M. Kati, “The psychological impact of cutaneous leishmaniasis," Clinical and Experimental Dermatology, vol. 29, no. 5, pp. 464-467, 2004. 

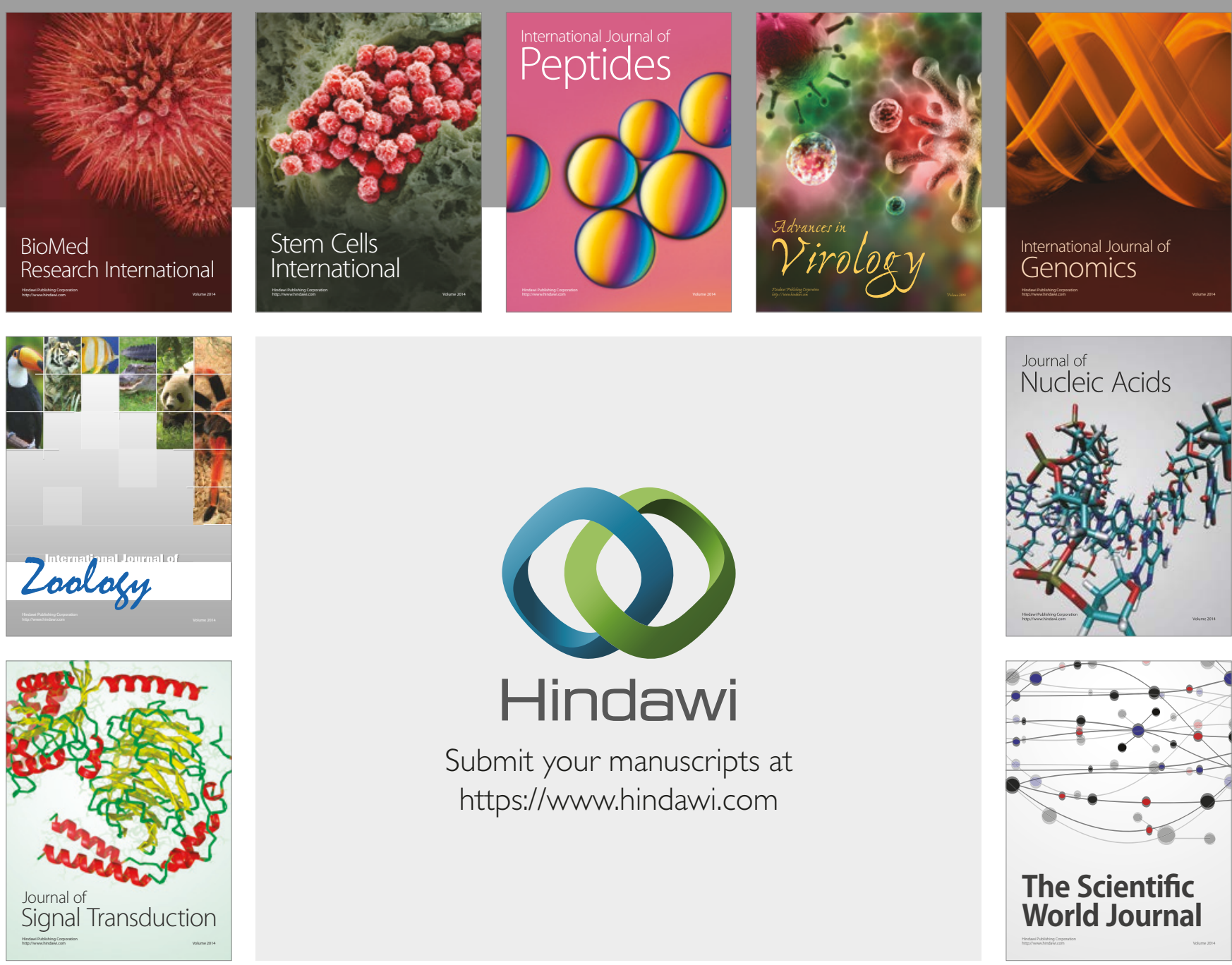

Submit your manuscripts at

https://www.hindawi.com
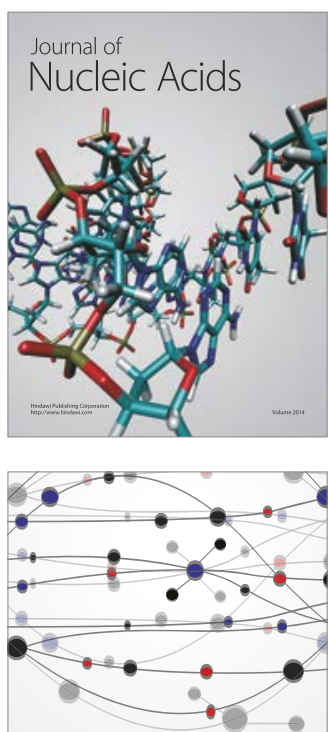

The Scientific World Journal

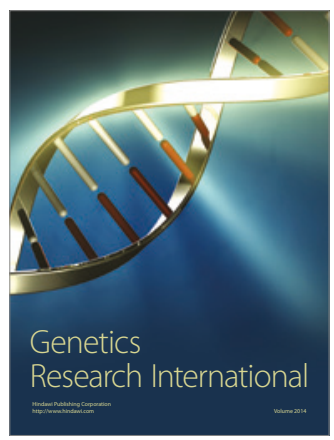

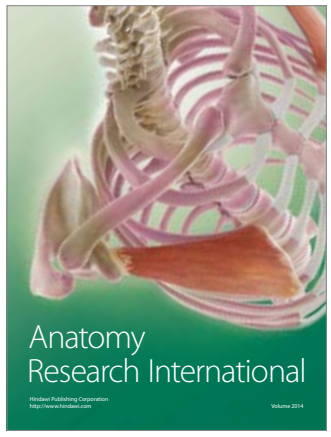

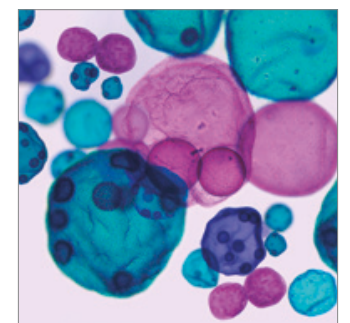

International Journal of Microbiology
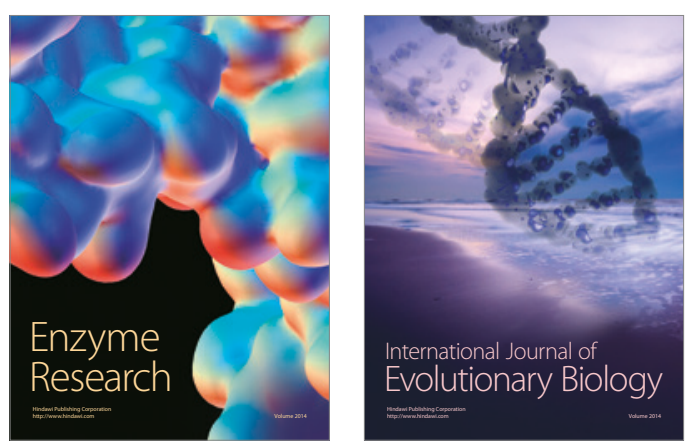
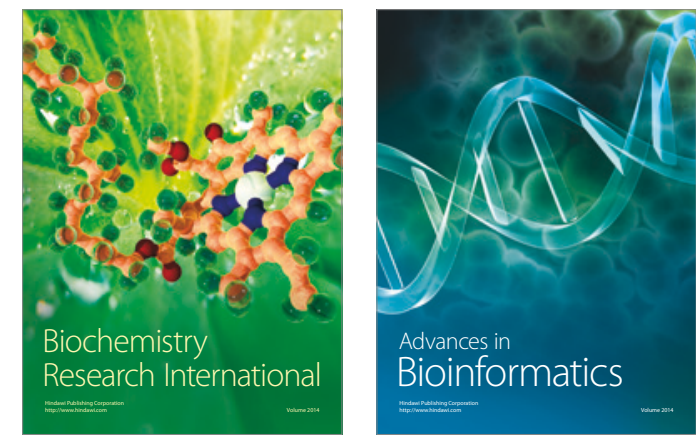

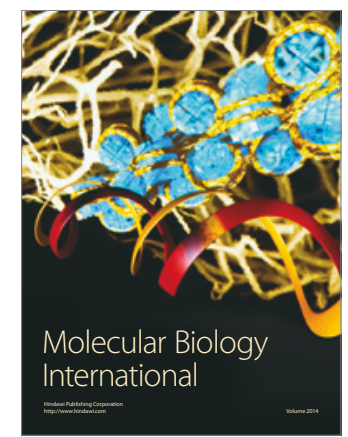

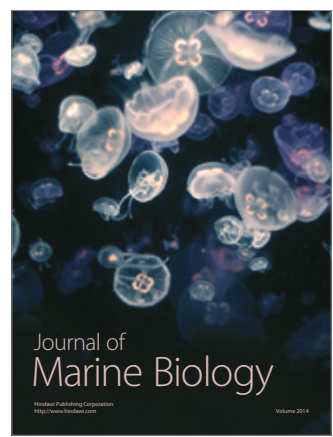

